\title{
Acute mesenteric ischaemia after cardiac surgery: clinical suspicion is key to survival
}

Singapore Med J 2020; 61(11): 613 https://doi.org/10.11622/smedj.2020153

Dear Sir,

Acute mesenteric ischaemia (AMI) post cardiac surgery is a challenging clinical problem that is often diagnosed late and carries a high mortality rate. Prompt diagnosis based on clinical suspicion is of paramount importance, as patients usually deteriorate abruptly. The decision to proceed with immediate diagnostic and therapeutic surgical interventions, without diagnostic imaging modality, is the key to survival. We herein report a case of AMI after coronary artery bypass grafting in a 69-year-old woman who underwent emergent diagnostic laparoscopy and bowel resection on clinical impression alone.

Our patient underwent coronary artery bypass grafting for left main coronary disease and triple vessel disease. She recovered well until the fourth postoperative day, when she was noted to be lethargic and tachycardic but remained in sinus rhythm without any documented atrial fibrillation. She became hypotensive within three hours refractory to crystalloid fluid boluses of $1 \mathrm{~L}$ in total. A bedside examination revealed otherwise unremarkable cardiovascular and respiratory systems, but the abdomen was tense and tender without guarding or rebound. Blood tests revealed a drop in haemoglobin level, from $11 \mathrm{~g} / \mathrm{dL}$ to $10 \mathrm{~g} / \mathrm{dL}$, and worsening renal function with a raised creatinine level from $70 \mathrm{mg} / \mathrm{dL}$ to $170 \mathrm{mg} / \mathrm{dL}$. A bedside echocardiogram revealed no pericardial effusion with preserved contractility. Despite increasing inotropic support, the patient remained hypotensive and became obtunded. She was intubated in the cardiothoracic intensive care unit and had a peri-intubation cardiac arrest, requiring resuscitation with return of spontaneous circulation.

Given the inconclusive abdominal examination findings, abrupt clinical deterioration and worsening lactic acidosis, an urgent surgical consultation was sought. The decision was made for urgent surgical exploration to exclude bowel ischaemia. Given the urgency of her condition, abdominal radiography was not done before the patient underwent a diagnostic laparotomy in the operating theatre. Intraoperative findings were significant for moderate amounts of haemorrhagic ascites and a long segment of ischaemic transverse colon (Fig. 1). This was then converted to a segmental colectomy with temporary abdominal closure. The patient recovered well thereafter and the acidosis improved. She was taken to the operating theatre two days later for relook laparotomy and the creation of an end colostomy. She had a slow recovery after the operation and was eventually discharged to a rehabilitation hospital 34 days after her original operation.

AMI after cardiopulmonary bypass for cardiac surgery has an incidence of $0.86 \%$ and a mortality rate of $67 \% .^{(1)}$ This high mortality rate is mostly attributed to delays in diagnosis or inappropriate clinical management. ${ }^{(2)}$ An intestinal ischaemia after cardiac surgery is most often due to splanchnic vasoconstriction that leads to low flow and regional ischaemia, which is called non-occlusive mesenteric ischaemia. It can be due to low cardiac output and may be aggravated by inotropic support and pre-existing atherosclerosis. ${ }^{(3)}$ Demographic and surgical variables that have been reported as risk factors for an intestinal ischaemia after cardiopulmonary bypass are arterial hypotension, postoperative heart failure, renal insufficiency, age $>70$ years, hypovolaemia, cardiopulmonary bypass time > 150 minutes, New York Heart Association

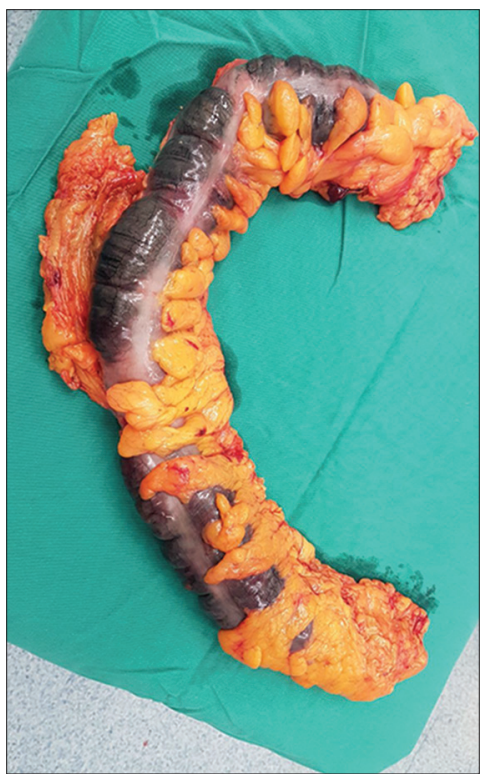

Fig. 1 Photograph shows the intraoperative specimen from the patient's diagnostic laparotomy, revealing a long segment of ischaemic transverse colon. Class III-IV, active smoking, sepsis, intra-aortic balloon pump support, use of vasopressors, and pre-existing atherosclerotic lesions.(4)

For our patient, hypovolaemia and pre-existing atherosclerotic lesions were the risk factors that predisposed her to an AMI. Given the degree of atherosclerotic disease within her coronary arteries, with over $90 \%$ of stenosis in all three major vessels, mesenteric vessels were also likely to have been affected. Any transient hypotension may have provoked a mesenteric ischaemic episode with subsequent bowel necrosis. Given the grave prognosis of such a complication, prompt surgical exploration is needed even in the absence of clinical findings. Survival is directly related to the degree of bowel ischaemia and the extent of bowel resection that is required.

Yours sincerely,

Evangelos Papadimas ${ }^{1}$, Giap Swee $\underline{K a n g}^{1}$

${ }^{1}$ Division of Cardiac Surgery, Department of Cardiac, Thoracic and Vascular Surgery, National University Heart Centre, Singapore. Evangelos.papadimas@gmail.com

\section{References}

1. Eris C, Yavuz S, Yalcinkaya S, et al. Acute mesenteric ischemia after cardiac surgery: an analysis of 52 patients. ScientificWorldjournal $2013 ; 2013: 631534$.

2. Merle C, Lepouse C, de Garine A, et al. Surgery for mesenteric infarction: prognostic factors associated with early death within 72 hours. J Cardiothorac Vasc Anesth 2004; $18: 734-41$.

3. Kazui T, Yamasaki M, Abe K, Watanabe S, Kawazoe K. Non-obstructive mesenteric ischemia: a potentially lethal complication after cardiovascular surgery: report of two cases. Ann Thorac Cardiovasc Surg 2012; 18:56-60.

4. Pang PY, Sin YK, Lim CH, Su JW, Chua YL. Outcome and survival analysis of intestinal ischaemia following cardiac surgery. Interact Cardiovasc Thorac Surg 2012; 15:215-8. 